\title{
Layer-by-layer Assembled Multilayer Membrane with Controllable Amount of Growth Factors for Osteogenic Differentiation
}

\author{
Zhuang Liangting, Zhinan Cao, Yumin Li, Yihong Gong* \\ Department of Biomedical Engineering, School of Engineering, Sun Yat-sen University, Guang- \\ zhou, 510006, P.R. China \\ Guangdong Provincial Key Laboratory of Sensor Technology and Biomedical Instrument ( Sun Yat- \\ sen Uni-versity) \\ * Corresponding author
}

Keywords: multilayer membrane, layer-by-layer self-assembly, osteogenic differentiation.

\begin{abstract}
Creating a bioactive cell-microenvironment and osteogenic functionality interface can be used for the surface modification of bone tissue engineering material, showing a good application prospect. In this paper, a bioactive and osteogenic functionality multilayer membrane with controllable amount of growth factors was fabricated by layer-by-layer assembly technique. Four biological macromolecules (casein, heparin, aidic fibroblast growth factor (aFGF) and collage I) were introduced onto the surface of polyurethane (PU) membrane. Fluores-cence intensity analysis and a toluidine blue assay revealed that the successfully multilayer membrane assembly process. The amount of aFGF loaded on membrane was regulated by changing the concentration of heparin, and aFGF can sustainably released from the membrane for 8 days. FDA staining and MTT assay showed that mesen-chymal stem cells (hMSCs) on growth factors multilayer membrane fully spread and displayed more obvious proliferation behavior. These preliminary results collectively show a practical and simple assembly technique, through which a bioactive and osteogenic material interface can be easily fabricated. This setup has the potential to be used for bone tissue engineering.
\end{abstract}

\section{Introduction}

Many biodegradable synthetic materials are paid more and more attentions for their use as scaffolds to support the regeneration of tissue-engineered organs such as cartilage, blood vessel, and skin. However, the intrinsic hydrophobic property of these polymers restricts their further applications. Hence, many surface modification methods are put forward to alter the surface properties of polymeric biomaterials, such as plasma treatment, ozone or photo induced grafting, surface oxidation and so on. However, these simple modification only mainly improve the hydrophilic of polymeric scaffold surfaces, but it may not be sufficient to allow the surface functionalization, such as osteogenic differentiation, which is vital important to activate the autologous regeneration mechanism [Hu Y et al.,2012].

Incorporation of growth factors is one of promising ways to improve the functionality and biocompatibility of tissue engineering materials. Growth factors are proteins secreted by cells and served as signaling agents for regulating proliferation and differentiation of different types of cells in the bone defect repairing process [Reiff DA et al.,2001]. Acidic fibroblast growth factor (aFGF) is isolated from bovine pituitary glands, belonging to the multifunctional proteins known as the fibroblast growth factor family (FGFs). Acidic fibroblast growth factors possess several functions including angiogenesis, promoting collateral circulation, inducing mitosis and differentiation, exhibiting an important role in skeletal development and postnatal osteogenesis [Marie P et al.,2003]. Successful use of aFGF has been notoriously difficult since they have half-lives only on the order of minutes.

Recently, layer-by-layer (LBL) assembly has been proposed by Decher et al and explored to form a bioactivity multilayers on material surface based on the alternate adsorption of polyelectro- 
lytes with opposite charge [He X et al.,2012, Tang Z et al.,2006]. Compared to other techniques, LBL assembly is a simple, inexpensive and powerful approach to introduce biological macromolecules to the surface of arbitrary solid substrates, which can be applied to functionalization of both two-dimension film and three-dimension scaffold. The most important advantage of this technique is that the incorporated macromolecules are stable and commendably retained its biological activity. Therefore, LBL technique is an ideal way to incorporate growth factors onto the surface of materials and creat an interface with good functionality and biocompatibility for tissue engineering. And this technique provide a more controllable manner for growth factors and growth factors can sustainably release from the membrane in a long period time.

In this paper, a bioactive and osteogenic functionality multilayer membrane with controllable amount of growth factors was fabricated by LBL assembly technique. Collagen I and aFGF were used as the cationic polyelectrolytes. Heparin and casein were used as the anionic one. Heparin is a glycosaminoglycan and has the ability to bind aFGF because of its strong negative charge. We explored that the amount of the incorporation of aFGF can be regulated by adjusting the heparin concentration and studied the release kinetic of aFGF from the multilayer membranes. Further, we evaluated the biocompatibility and osteogenic functionality of aFGF multilayer membrane. And this study may make a significant sense to the surface modification of biodegradable tissue regeneration scaffolds.

\section{METHODS}

\section{Preparation polyelectrolyte solution and construction aFGF multilayer by LBL self- assembly}

HEPES solution were prepared by dissolve $2.383 \mathrm{~g}$ hepes and $8.770 \mathrm{~g} \mathrm{NaCl}$ in $1000 \mathrm{ml}$ water $(\mathrm{Ph}=7.0)$. Negative polyelectrolyte solution was the mix solution of $0.2 \mathrm{mg} / \mathrm{ml}$ collagen I and 1 $\mathrm{mg} / \mathrm{ml}$ aFGF (COLI/aFGF). Positive polyelectrolyte solutions were made by casein and heparin in HEPES solution, dividing into three groups by fixing casein at $2 \mathrm{mg} / \mathrm{ml}$ and heparin with a dose range of $4 \mathrm{mg} / \mathrm{ml}, 0.4 \mathrm{mg} / \mathrm{ml}$ and $0.04 \mathrm{mg} / \mathrm{ml}$ (CA/HEP).

PU membranes were fabricated and aminolyzed as the method previously described [Zhu Y et al., 2003]. Acidic fibroblast growth factor multilayer membranes were manufactured on aminolyzed PU membrane by accomplishing four steps: (1) PU membranes were treated in CA/HEP solution for 15min; (2) rinsed with HEPES three times; (3) immersion in aFGF/COLI solution for 15min; (4) washed a large amount of HEPES. Repeat the four steps until the desired layers of ((CA/HEP)/(aFGF/COLI))n multilayer membranes were obtained. The final substrates assembled with different dose of heparin were termed "LBL-HEP_4", "LBL-HEP_0.4" and "LBL-HEP_0.04". "LBL-CA/COLI" was denoted to represent the sample only assembled with casein and collagen I.

\section{Characterization of the aFGF multilayers}

To verify our hypothesis that the heparin concentration has a positive effect on its amount in self-assemble process, the amount of immobilized heparin was determined by a toluidine blue assay after deposition each layer of CA/HEP. The assembled PU membrane was immersed into toluidine blue solution and put in a shaker for 30min. Then add $500 \mu \mathrm{l}$ hexane to the mix solution and disperse phase can been saw after shaken up. The absorbance at $631 \mathrm{~nm}$ of the aqueous phase liquid was measured by a microplate reader (BioTek synergyth4, USA). The amount of heparin can be calculated according to the heparin standard curve, constructed based on using a series of heparin concentrations, after absorbing each layer of CA/HEP. Further, to investigate if the amount of aFGF introduced onto the surface of PU membrane has a certain relationship with the amount of immobilized heparin, the fluorescence spectra of rhodamine-labeled aFGF were measured during the LBL self assemble process by a microplate reader. The fluorescence spectra of FITC-labeled casein (FITC-caesin) were also conducted to reveal the relationship between assembling components. The static water contact angle was detected to evaluate the surface wettability after each polyelectrolyte deposition by using a DSA100 system (Kruss, Hamburg, Germany). 


\section{In vitro aFGF release study}

The aFGF multilayers membrane were fabricated, using rhodamine-labeled (RD-aFGF) instead of aFGF. Then membranes were soaked in $1 \mathrm{ml}$ PBS (pH 7.4) and put into a $0.5 \% 37{ }^{\circ} \mathrm{C}$ incubator to explore aFGF release kinetics. The supernatants were collected and substituted by fresh PBS at particular time intervals of 0.5 h, 1 h, 2 h, 4 h, 8 h, 12 h, 18 h and 24h, 72 h (3 days), 144 h ( 6 days), $192 \mathrm{~h}$ (8 days). The fluorescence intensity of samples was measured by a microplate reader at excitation of $560 \mathrm{~nm}$ and emission of $585 \mathrm{~nm}$. The amount of aFGF releasing from the films were calculated according to the RD-aFGF fluorescence standard curve.

\section{Morphology and proliferation of hMSCs}

hMSCs were derived from bone marrow aspirates of healthy volunteers as previously described and cultured in basal growth medium: alpha-modified Eagle's medium (a-MEM; thermo, USA) supplemented with $10 \%$ fetal bovine serum, $100 \mathrm{U} / \mathrm{ml}$ penicillin, $100 \mu \mathrm{g} / \mathrm{ml}$ streptomycin at $37^{\circ} \mathrm{C}$ with 5\% CO2. Passage 3 of hMSCs were seeded onto LBL-HEP_4, LBL-HEP_0.4, LBL-HEP_0.04, LBL-CA/COLI, as-received PU membrane and blank wells at the density of 3000 cells/cm2 in 24well plate. Cells were also seeded onto the positive control group: LBL- CA/COLI substrates with aFGF directly added in a medium (named "LBL-CA/COLI-aFGF"). At predetermined time interval, 3-[4,5-dimethylthiazol-2-yl]-2,5-diphenyltetrazolium bromide (MTT) assay was used to study cell proliferation on the sample. In brief, $0.5 \mathrm{ml}, 0.5 \mathrm{mg} / \mathrm{ml}$ MTT/PBS solution was added into each well and incubated for 4 hour in a $37{ }^{\circ} \mathrm{C}, 5 \% \mathrm{CO} 2$ incubator. Then $300 \mu$ dimethyl sulfoxide (DMSO; Sigma) was added and incubated for $5 \mathrm{~min}$. The absorbance at $570 \mathrm{~nm}$ of the solution was measured by a microplate reader. In order to observe cell morphology on aFGF multilayer membranes, hMSCs were stained with fluorescein diacetate (FDA, $5 \mu \mathrm{g} / \mathrm{ml}$ ). Living cells were visualized as green under a DMLB fluorescence microscope (Olympus, Japan).

\section{Results and analysis}

\section{Characterization of the aFGF multilayers}

The amount of immobilized heparin in the LBL-deposition process was determined by a toluidine blue assay.
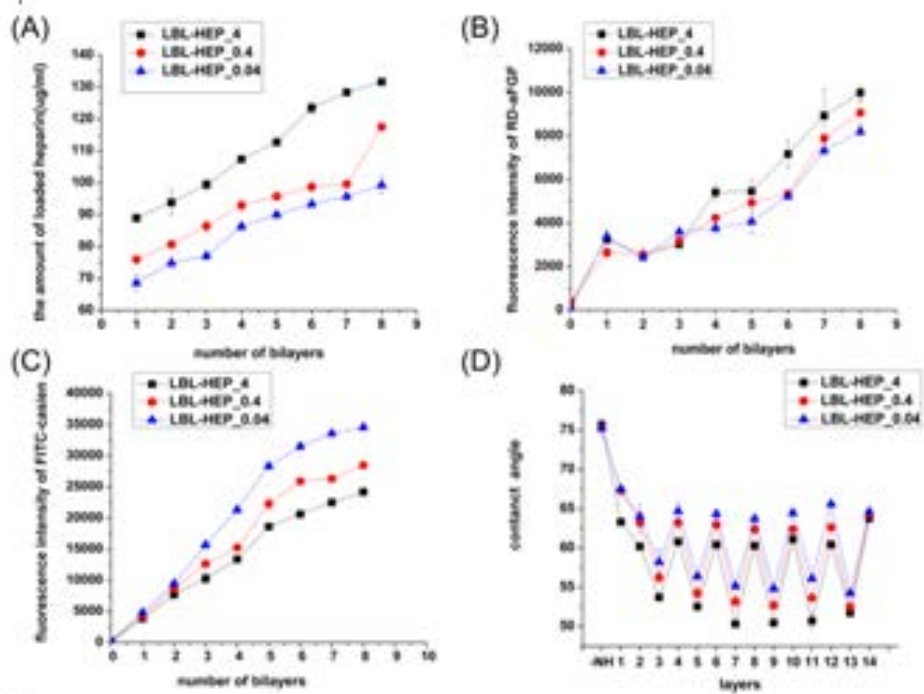

Figure 1. Monitoring and analysis the Construction of aFGF multilayers membranes: (A) toluidineblue assay;(B) fluorescence spectra of RD-aFGF; (C) fluorescence spectra of FITC- casein;(D) water contact angle.

As we supposed, Figure 1A visually displayed the amount of heparin increased linearly along with the number of bilayers, which declared that aFGF membranes were fabricated successfully. The amount of heparin assembled into aFGF membranes increased as the increasement with heparin concentration. After each deposition of CA/HEP, the amount of heparin incorporated in LBL- 
HEP_4 was always larger than that of heparin incorporated in LBL-HEP_0.4, while the amount of heparin in LBL-HEP_0.04 was the least. Besides, we found that the amount of heparin of first deposition was significant great probably due to the rough surface of the aminolyzed PU with a large amount of nanometers diameter, which made it more surface area for heparin assemble compare to other layer. Further, fluorescence spectra analysis was used to study the relationship between assembling components. One of the normal aFGF, casein was replaced by a RD-aFGF or FITC-casein in each LBL assembly. As shown in Figure 1B, the fluorescence intensity of all multilayers was increasing proportionally with the layer number. The higher heparin concentration was, the stronger fluorescence was, declaring that more aFGF were introduced on the surface of PU membranes, which can be ascribed to the specific binding between heparin and aFGF. Heparin possessed a competitive relationship with casein because they all used as negative polyelectrolyte in the self assembly. Hence, when the PU substrate was immersed in higher concentration heparin solution, more heparin were incorporated, while less casein were incorporated (Figure $1 \mathrm{C}$ ).

Figure 1D shows the alternation of water contact angle as a function of layer number. Before absorbed any protein , the water contact angles of the aminolyzed PU was determined to be $75.2^{\circ}$ while it decreased to $63 \sim 67^{\circ}$ after the first layer of CA/HEP, which illustrated that a self-assembled monolayer can change surface wettability. Basically, the static water contact angles oscillated be-

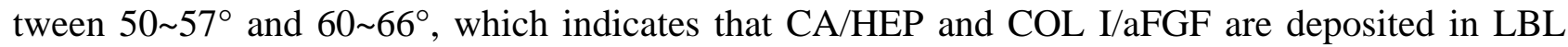
manner. As the same outmost layer, different heparin concentration presented different contact angle. The higher heparin concentration was, the larger the contact angle was. It has a lot to do with the strong electronegative of heparin.

\section{In vitro aFGF release study}

To investigate the release kinetics of aFGF, we detected the cumulative release amount of aFGF from multilayers. As Figure 2 shown, a burst release was observed at initial $8 \mathrm{~h}$, and then the remained aFGF released steadily and slowly until day 8. It was mainly attributed to the fact that a layer of aFGF as the outmost component was directly exposed to PBS and released fast. Then, the additional aFGF inside the multilayer released slowly because the initial polyelectrolyte membranes were stable, relatively. The released curves exhibited sustained release of different concentrations of aFGF according to the amount of aFGF contained in the multilayer membranes, which was mainly decided by the assembled heparin concentration.

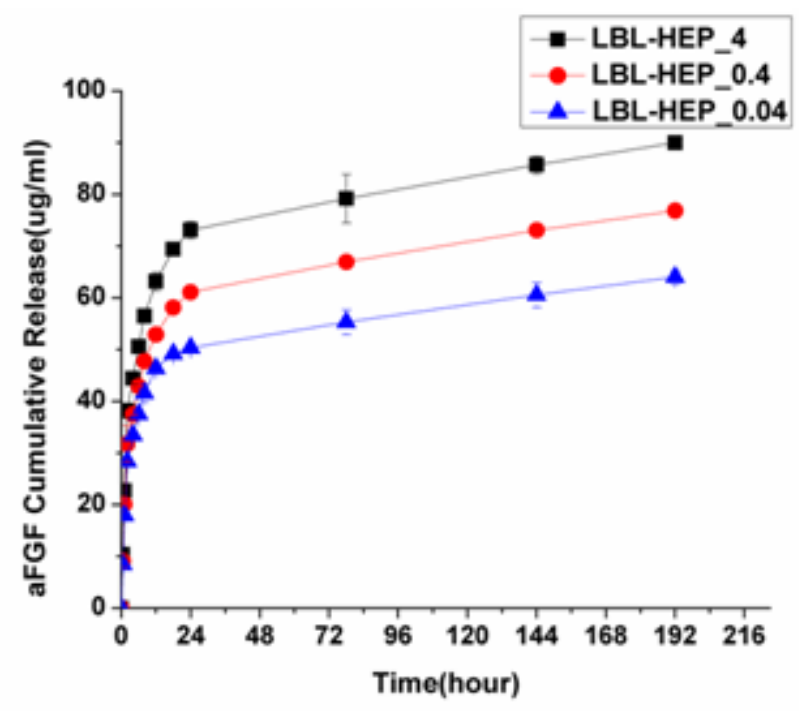

Figure 2. In vitro release of aFGF from LBL-HEP_4, LBL-HEP_0.4 and LBL-HEP_0.04.

\section{The cytocompatibility of aFGF multilayers membranes}

As shown in Figure 3, plenty of HMSCs significantly connected and overlapped on aFGF membranes, displaying normal elongated morphology, which was similar to the HMSCs grown on the positive control group: LBL-CA/COLI-aFGF, while only a few HMSCs were adhered to unmodified PU and TCPS and were not spread completely. 
Compared to unmodified PU and LBL-CA/COLI, the cells exhibited more noticeable proliferation on aFGF membranes during 14-d culture (Figure 4). In aFGF membrane groups, the membranes LBL-HEP_0.4 had the largest amount of cells, which were closed to the positive control, while HMSCs on LBL-HEP_4 were statistically more than that on LBL-HEP_0.04, just as supposed HMSCs' proliferation had something to do with the amount of aFGF released from the membranes.
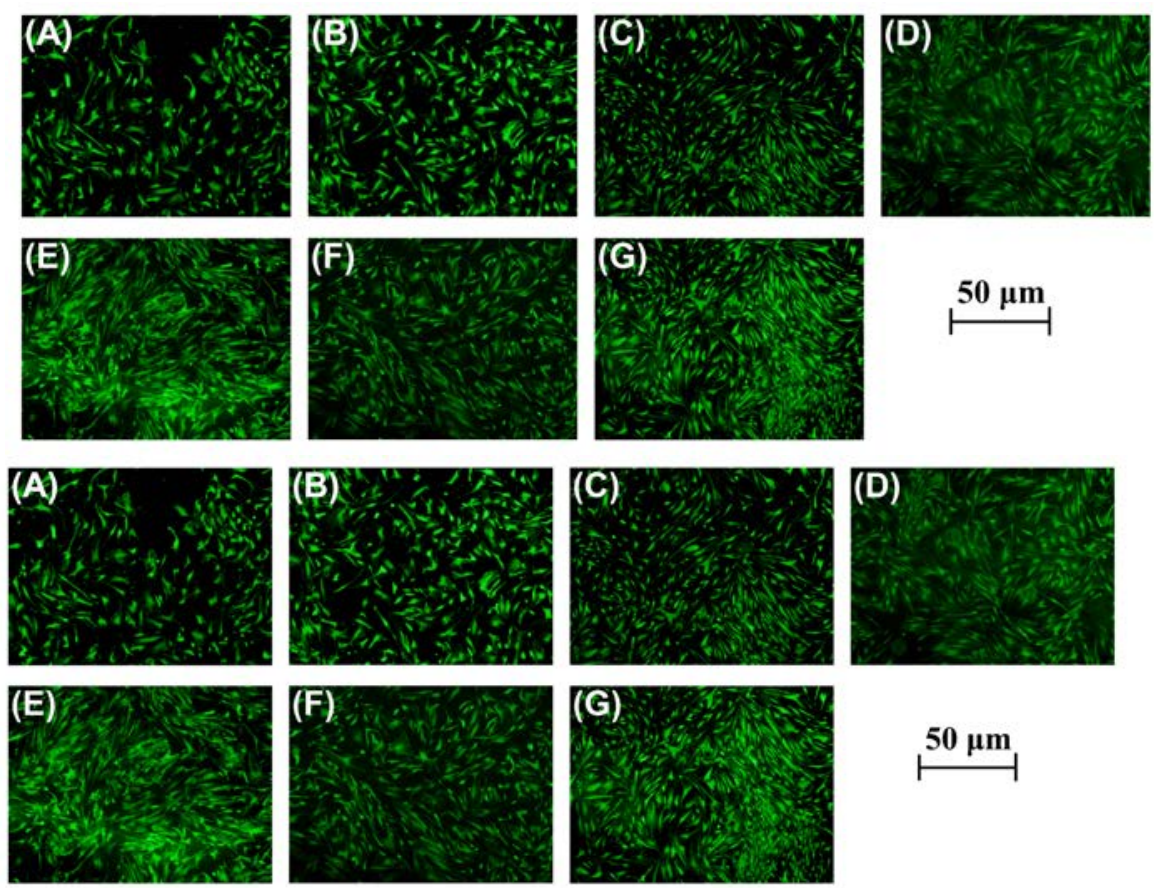

Figure 3. FDA staining of HMSCs cultured on different specimens at day 5.

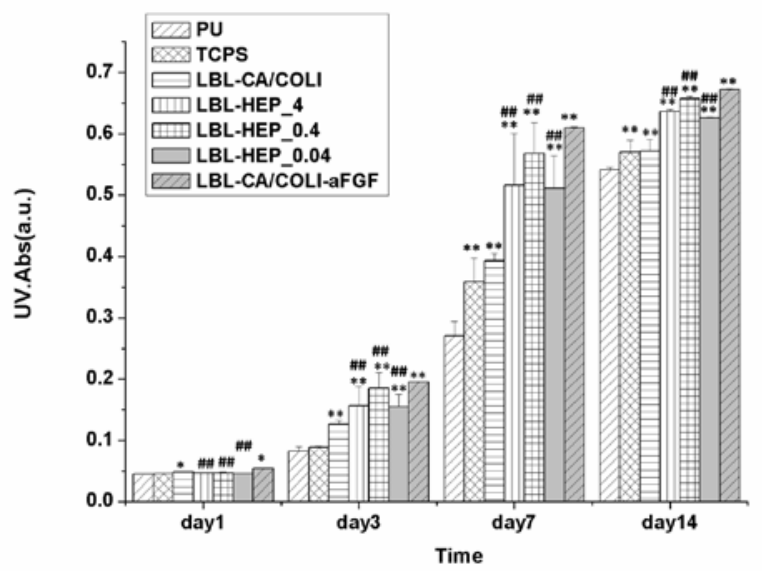

Figure 4. The hMSCs proliferation was performed by MTT assay.

\section{Conclusion}

The bioactive aFGF has been successfully constructed onto the surfaces of PU membranes via a LBL assembly manner. The amount of aFGF incorporated into the membranes can be regulated by altering the assembled heparin concentration. Biological assay revealed aFGF multilayer membranes can significantly improve the cytocompatibility of PU membranes. aFGF released from polyelectrolyte membranes facilitated the proliferation of osteoblast and the improvements exhibited a higher value with larger does of aFGF except for LBL-HEP_4 groups, which was probably due to more hydrophobic heparin in it. The in vitro aFGF release study showed that the release of the incorporated aFGF occurred mostly during the first $24 \mathrm{~h}$, in which osteoblasts were mainly in a 
multiplication phase. Afterwards, the residual aFGF can continuously be free for up to 8 days and accelerated the cells proliferating in a rather long period. It has been found that cell adhesion plays an important role in the regulation of successive cell functions including proliferation, migration and differentiation. It was confirmed that aFGF multilayer membranes promoted cell attachment dramatically via the expression of vinculin in cell.

In summary, we fabricate a bioactive and osteoinductive mutilayer membrane with controllable amount of growth factors by a practical and simple LBL technique. And this surface modification can apply to biodegradable tissue regeneration scaffolds and show a good application prospect.

\section{Acknowledgement}

This work was supported by the National Natural Science Foundation of China (No.51103182, No.51203194); Science and Technology Program of Guangzhou (2060204, 201300000057), Science and Technology Planning Project of Guangdong Province (No. 2011A060901013), Guangdong Innovative Research Team Program (No.2009010057).

\section{References}

1. Hu Y et al. 2012. Regulation of the differentiation of mesenchymal stem cells $<\mathrm{i}>$ in vitro $</ \mathrm{i}>$ and osteogenesis $<\mathrm{i}>$ in vivo $</ \mathrm{i}>$ by microenvironmental modification of titanium alloy surfaces. Biomaterials 33: 3515-28

2. Reiff DA et al. 2001. Acidic fibroblast growth factor attenuates the cytotoxic effects of peroxynitrite in primary human osteoblast precursors. Journal of Trauma-Injury, Infection, and Critical Care 50: 433-9

3. Marie P. 2003. Fibroblast growth factor signaling controlling osteoblast differentiation. Gene 316: 23-32

4. He X et al. 2012. Layer-by-layer assembly of type I collagen and chondroitin sulfate on aminolyzed PU for potential cartilage tissue engineering application. Appl Surf Sci 258: 9918-25

5. Tang Z et al. 2006. Biomedical Applications of Layer - by - Layer Assembly: From Biomimetics to Tissue Engineering. Adv Mater 18: 3203-24

6. Zhu Y et al. 2003. Layer-by-Layer assembly to modify poly (L-lactic acid) surface toward improving its cytocompatibility to human endothelial cells. Biomacromolecules 4:446-52. 